\title{
Threat-sensitive responses to disturbance cues in juvenile convict cichlids and rainbow trout
}

\author{
Meaghan A. Vavrek \& Grant E. Brown*
}

Department of Biology, Concordia University, 7141 Sherbrooke St. West, Montreal, Qc, H4B 1R6, Canada (*e-mail: gbrown@alcor.concordia.ca)

Received 31 Jan. 2008, revised version received 4 Apr. 2008, accepted 19 Apr. 2008

Vavrek, M. A. \& Brown, G. E. 2009: Threat-sensitive responses to disturbance cues in juvenile convict cichlids and rainbow trout. — Ann. Zool. Fennici 46: 171-180.

We conducted laboratory experiments (1) to confirm the existence of a non-injury released disturbance cue in juvenile convict cichlids and rainbow trout, and (2) to determine if cichlids and trout exhibit a graded threat-sensitive antipredator response to varying concentrations of disturbance cues. The results of our first experiment demonstrate that both cichlids and trout exhibit significant antipredator responses (reductions in time spent moving, foraging rate and area use) to the odour of conspecifics that had been exposed to a realistic predator model but not to the odour of undisturbed conspecifics. The results of our second experiment demonstrate that cichlids and trout exhibit reduced time spent moving and foraging rates proportional to the concentration of disturbance cue detected. Together, these results confirm the presence of disturbance cues in cichlids and trout and demonstrate that disturbance cues provide sufficient information to allow for graded threat-sensitive responses.

\section{Introduction}

The predator avoidance patterns of most prey species are shaped by a series of trade-offs between the benefits associated with successful detection and avoidance of predation threats and those associated with a suite of other fitness related behaviours such as foraging, mating and territorial defence (Godin \& Smith 1988, Lima \& Dill 1990). Therefore, in order to maximize potential fitness benefits, prey individuals should respond in an appropriate manner to the current level of risk; exhibiting low intensity anti-predator behaviour in response to low levels of risk and proportionally more intense responses as perceived risk levels increase (Helfman 1989, Lima \& Dill 1990, Foam et al. 2005). Such a trade-off is referred to as threat-sensitivity (Helfman 1989, Brown et al. 2006) and the ability to make threat-sensitive behavioural decisions assumes that prey can reliably assess local predation threats (Brown et al. 2006).

Within aquatic ecosystems, prey organisms rely heavily on chemosensory information to detect nearby predation threats (Chivers \& Smith 1998, Kats \& Dill 1998, Wisenden 2000). The most widely studied of these are the damagereleased chemical alarm cues. Such cues are typically localized in the epidermis and are released following mechanical damage to the skin as would occur during a predation event (Chivers \& Smith 1998). When detected by nearby conspecifics and some sympatric heterospecifics, damage-released cues may elicit a dramatic, short-term change in 
species-typical antipredator behaviour (Chivers \& Smith 1998, Brown 2003). Several researchers have recently demonstrated that the concentration of alarm cues detected provides useful information to cue receivers about the relative threat of predation, allowing for threat-sensitive predator avoidance patterns. Prey fish may exhibit antipredator behaviour at an intensity proportional to the concentration of alarm cue detected (i.e., a graded response pattern, Jachner \& Rydz 2002, Dupuch et al. 2004, Ferrari et al. 2005, Brown et al. 2006). Alternatively, prey may exhibit a nongraded or hypersensitive response pattern (Helfman \& Winkleman 1997), characterized by high intensity behavioural responses above some minimum threshold (Marcus \& Brown 2003, Brown et al. 2006, Blanchett et al. 2007). Both graded and hypersensitive threat-sensitive response patterns are argued to allow prey individuals to balance the conflicting demands of successful predator avoidance and other fitness related behaviours (Helfman 1989, Helfman \& Winkleman 1997, Brown et al. 2006).

Another form of chemosensory information, which has been far less studied, are the disturbance cues (Wisenden 2000). Disturbance cues are released by stressed or disturbed prey prior to an attack by a predator (Wisenden et al. 1995, Jordão \& Volpato 2000), either in the urine or diffused across the gills. As such, these cues do not require any mechanical damage by predators in order to be released. Disturbance cues have been demonstrated in a variety of taxonomically diverse aquatic prey species, including invertebrates (crayfish, Oronectes virilis: Hazlett 1990a; hermit crabs, Calcinus laevimanus: Hazlett 1990b; red sea urchins, Strongylocentrotus franciscanus: Nishizaki \& Ackerman 2005), amphibian (red-legged frogs, Rana aurora: Kiesecker et al. 1999) and freshwater prey fishes (Iowa darters, Etheostoma exile: Wisdenden et al. 1995; slimy sculpins, Cottus cognatus: Bryer et al. 2001; brook trout, Salvelinus fontinalis: Mirza \& Chivers 2001a (though see Mirza \& Chivers 2000); pacus, Praractus mesopotamicus: Jordão \& Volpato 2000; and convict cichlids, Archocentrus nigrofasciatus: Jordão 2004).

Within prey fishes, the response to disturbance cues is thought to represent a lower level of predation risk as compared with other chemo- sensory or visual predator cues (Wisenden et al. 1995, Bryer et al. 2001, Jordão 2004). As such, it remains unknown if disturbance cues provide sufficient risk assessment information to allow for graded threat-sensitive response patterns, as seen for damage-released alarm cues (Brown et al. 2006). The purpose of our current study was twofold. Firstly, we exposed juvenile convict cichlids and rainbow trout (Oncorhynchus mykiss) to putative disturbance cues (versus the odour of undisturbed conspecifics) to verify the presence of disturbance cues. Only a single salmonid has been tested for the response to disturbance cues (brook trout), with mixed results (Mirza \& Chivers 2000, 2001a). The single study with convict cichlids suggests only a weak response to disturbance cues (Jordão 2004). Secondly, we exposed cichlids and trout to varying concentrations of a standard disturbance cue solution (versus a distilled water control) to test the hypothesis that the response intensity is proportional to the concentration detected (i.e. graded).

\section{Methods}

Juvenile convict cichilds were obtained from our laboratory stock population. These fish were descendants from laboratory crosses made approximately four generations previously from laboratory stock and wild caught cichlids from Costa Rica. Prior to testing, cichlids were held in 110-1 glass aquaria, filled with continuously filtered dechlorinated tap water $\left(26^{\circ} \mathrm{C}, \mathrm{pH} \sim 7.2\right.$, 12:12 light:dark cycle) and a gravel substrate. Cichlids were fed ad libitum, twice daily, with commercial flake food and brine shrimp (Artemia spp.). Juvenile rainbow trout were obtained from a commercial hatchery (Pisciculture des Arpents Verts, Ste. Edwidge de Clifton, Quebec). Prior to testing, trout were held in 390-1 recirculating tanks, with a continuous supply of dechlorinated tap water $\left.(\sim 750 \mathrm{ml} \mathrm{min})^{-1}\right)$ at $18{ }^{\circ} \mathrm{C}(\mathrm{pH} \sim 7.0$, 12:12 light:dark cycle) . Trout were fed ad libitum, twice daily, with commercial trout chow.

\section{Stimulus collection}

To collect disturbance cues (experimental) and 
the odour of undisturbed conspecifics (controls), we placed donor fish (see below) into 20-1 glass aquaria, filled with 151 of dechlorinated tap water at similar temperature and $\mathrm{pH}$ conditions as the holding tanks 24 hours prior to stimulus collection. Stimulus tanks contained a charcoal corner filter. One hour prior to stimulus collection, we turned the filter off. For the disturbance treatments, we slowly passed a realistic fish predator model $(15 \mathrm{~cm})$ attached to a glass rod through the tank 20 times. We took care not to contact donor fish to avoid potentially releasing damage-released chemical alarm cues into the tank. Qualitative observations suggest that the donor fish did indeed react to the model predator with a strong antipredator response (dropping to the substrate and shoaling in a corner of the tank). Following model presentations, we waited 60 seconds, gently mixed the water and removed approximately $200 \mathrm{ml}$ of tank water to be used as a disturbance cue. For the odour of undisturbed conspecifics, we turned the filter off, as above, and removed a similar volume of water. In all cases, experimental and control stimuli were collected 'fresh' for each day of trials and were used within one hour of collection. Donors were used only once and returned to the stock population.

\section{Test tanks}

For both Experiments 1 and 2, test tanks consisted of a series of 37-1 glass aquaria, equipped with a gravel substrate and a single airstone. Test tanks were not filtered. In order to deliver stimuli without disturbing test fish, we attached $1.5 \mathrm{~m}$ of air line tubing to each tank, terminating immediately above the airstone. Tanks were wrapped in black plastic to prevent visual cues being transmitted between test fish. In addition, we divided test tanks into three vertical sections by drawing horizontal lines on the exterior of the tanks in order to facilitate area use (see below). In all cases, test fish were transferred to test tanks 24 hours prior to testing to allow sufficient time to acclimate. For both cichlids and trout, cue donors and test fish were taken from the same stock population. Fish were used once, either for donors or test fish, but never both. Both cichlids and trout were fed ad libitum up to
1 hour before testing. In all cases, there was food (flakes for cichlids, pellets for trout) remaining on the substrate during observations, allowing us to quantify foraging attempts without presenting additional food during trials (sensu Brown et al. 2006). For cichlids, we also added $0.5 \mathrm{~g}$ (in $5 \mathrm{ml}$ of tank water) of brine shrimp (Artemia spp.) before pre- and post-stimulus observation periods (see below) to ensure that the focal fish were active. We did not provide a food stimulus to the trout.

\section{Experimental protocol}

Trials consisted of a five-minute pre-stimulus and a five-minute post-stimulus observation period. Prior to an observation, we withdrew and discarded $60 \mathrm{ml}$ of tank water through the stimulus injection tube. We then withdrew (and retained) an additional $60 \mathrm{ml}$ of tank water. Following the pre-stimulus observation, we introduced $10 \mathrm{ml}$ of the required stimulus (see below) and slowly flushed it into the test tank using the retained tank water. For cichlid trials, we tested pairs of juvenile cichlids, as singleton cichlids are generally inactive and do not exhibit threat-sensitive antipredator responses (Brown et al. 2006). We scored behavioural measures for both test fish and used the mean values as the replicate datum. For trout trials, we tested individual rainbow trout. All trials were videotaped and scored. The order of trials was randomized within experiments for cichlids and trout.

\section{Experiment 1: Response to 'disturbance cues'}

The goal of this study was to confirm the antipredator response to disturbance cues in juvenile convict cichlids and rainbow trout and to determine if donor group size influenced the overall intensity of the response.

\section{Convict cichilds}

We collected disturbance cues and the odour of undisturbed conspecifics from groups of 5 or 
10 donors. Mean $( \pm$ SD) size of donors $=4.00 \pm$ $0.75 \mathrm{~g}$ and $4.40 \pm 0.57 \mathrm{~cm}$ standard length (SL). For both pre- and post-stimulus observation periods, we recorded foraging rate (number of foraging attempts per minute), time spent moving and area use. Area use was scored as the position of each test fish in the tank every 15 seconds. Area use scores ranged from 2 (both fish in the bottom third of the tank) to 6 (both fish in the top third of the tank). We defined foraging as pecking at the substrate, with the body at an angle greater than $45^{\circ}$ relative to the substrate (Grant et al. 2002). Reduced time moving, foraging rate and area use are indicative of an antipredator response in juvenile convict cichlids (Brown et al. 2006). Mean size of test fish at time of testing was 3.46 $\pm 0.67 \mathrm{~cm}$. We calculated the change in each behavioural measure by subtracting the poststimulus observation from the pre-stimulus value and used these difference scores as dependent variables in all analyses. We tested for an overall effect of disturbance cue and donor number using a MANOVA. We conducted a total of 15 replicates per treatment combination and individual cichlids were tested only once.

\section{Rainbow trout}

Trials were conducted as described above for cichilds expect that we tested individual trout and we recorded time (in seconds) in the bottom third of the tank rather than area use scores. Mean $( \pm \mathrm{SD})$ size of donors was $1.10 \pm 0.47 \mathrm{~g}$ and $3.69 \pm 0.35 \mathrm{~cm}$ SL. As with cichlids, we collected disturbance cues and the odour of undisturbed conspecifics from groups of 5 and 10 donors. Mean ( \pm SD) of trout at time of testing was $3.86 \pm 0.35 \mathrm{~cm} \mathrm{SL}$. We conducted a total of 15 replicates per treatment combination and individual trout were tested only once. Data were analyzed as above.

\section{Experiment 2: Response to varying concentrations of disturbance cues.}

The goal of this experiment was to determine if juvenile convict cichlids and rainbow trout respond to decreasing concentrations of distur- bance cues in a threat-sensitive (i.e. graded) fashion. For both cichlids (10 donors) and trout (5 donors), we generated a 'stock' disturbance cue solution and diluted it to the required relative concentration with distilled water. The experimental protocol was as described above, except we presented $10 \mathrm{ml}$ of disturbance cues at one of four concentrations (undiluted: $100 \%$; or diluted to $75 \%, 50 \%$ or $25 \%$ with the addition of distilled water) or a control of distilled water $(0 \%)$.

\section{Convict cichlids}

For both pre- and post-stimulus observations, we quantified time spent moving and frequency of foraging attempts as described above. We conducted a total of 30 replicates for each stimulus concentration, except for the $100 \%$ treatment where the video images were of poor quality $(N$ =29). We calculated the change in each behaviour measure (as above) and included these as dependent variables in a MANOVA. We used a priori polynomial contrasts to test the prediction that the intensity of antipredator responses should decrease proportionally with lower concentrations of disturbance cues. Mean size of donors was $4.14 \pm 0.67 \mathrm{~g}$ and $4.59 \pm 1.07 \mathrm{~cm} \mathrm{SL}$. Mean size of test fish was $3.49 \pm 0.06 \mathrm{~cm} \mathrm{SL}$.

\section{Rainbow trout}

As above, we recorded time spent moving and frequency of foraging attempts for individual trout exposed to disturbance cues at varying concentrations or a distilled water control. Data were analyzed as above. We conducted a total of 15 replicates for each stimulus concentration. Mean size of donors was $1.25 \pm 0.38 \mathrm{~g}$ and $3.89 \pm 0.18 \mathrm{~cm}$ SL. Mean size of test fish was $3.74 \pm 0.21 \mathrm{~cm} \mathrm{SL}$.

\section{Results}

\section{Experiment 1}

For convict cichlids, we found significant main effects of both donor number and disturbance 
cues, as well as a significant interaction between the two main effects (Table 1 and Fig. 1). There was no difference in antipredator response (odour of undisturbed vs. disturbed conspecifics) when 5 donors were used, but we observed a significant increase in antipredator behaviour when 10 donors were used. For rainbow trout, we found a significant effect of disturbance cue (Table 1 and Fig. 2), but no effect of donor group size or a significant interaction (Table 1 and Fig. 2).

\section{Experiment 2}

We found a significant overall effect of disturbance cue concentration for convict cichlids (MANOVA: $F_{4,144}=5.77, P<0.001$; Fig. 3 ). Our planned contrasts analysis revealed that for both foraging rate and time spent moving, there were significant univariate effects $\left(F_{4.144}=3.62, P=\right.$ 0.008 and $F_{4,144}=3.20, P=0.015$, respectively). The response pattern for both foraging rate and time moving are best described as linear versus quadratic contrasts (Table 2 and Fig. 3), suggesting that the decrease in response intensity with decreasing concentration is indeed graded.

Likewise, we found similar overall results for rainbow trout (MANOVA: $F_{4,75}=4.52, P=$ 0.003; Fig. 4). Both foraging rate and time spent moving exhibited significant univariate effects $\left(F_{4,75}=3.83, P=0.007\right.$ and $F_{4,75}=3.17, P=0.018$, respectively). As above, the response pattern for foraging and time moving are best described by the linear contrast terms (Table 2 and Fig. 4), demonstrating graded response patterns.

\section{Discussion}

Our results confirm the existence of early release disturbance cues in juvenile convict cichlids and rainbow trout. Previous work examining disturbance cues in convict cichlids found no reduction in foraging or time spent near a foraging patch or a refuge patch (Jordão 2004). However, Jordão (2004) did find an increase in area use, attributed to an increase in exploratory or predator inspection behaviour. This apparent discrepency is likely due to the experimental design used by Jordão (2004). In her experiment, cichlids were tested repeately over a five day period to a variety of treatments, receiving the disturbance cues of a single donor only on day 5. In our experiment, focal fish were tested only once. Likewise, previous work with juvenile brook charr have demonstrated mixed results, with Mirza and Chivers (2000) failing to find a response to disturbance cues while Mirza and Chivers (2001a) reporting positive results. Thus, the results of experiment 1 confirm the existence of disturbance cues in convict cichlids and extend the salmonid results to a second species.

Moreover, our results demonstrate that the relative concentration of disturbance cue detected provides useful information regarding the nature of local predation threats. Both cichilds and trout exhibited antipredator responses proportional to the concentration of disturbance cues detected. Jordão (2004) found only weak responses by convict cichlids exposed to the disturbance cues of singleton donors (presumably a much lower concentration that we used), further supporting a

Table 1. MANOVA results (Experiment 1) for convict cichlids and rainbow trout exposed to disturbance cues (versus the odour of undisturbed conspecifics) collected from 5 versus 10 conspecific donors. $N=15$ for each treatment combination.

\begin{tabular}{lrcl}
\hline & $F$ & df & $P$ \\
\hline Convict cichlids & & & \\
$\quad$ Donor number & 4.13 & 3,54 & $\mathbf{0 . 0 1}$ \\
$\quad$ Disturbance cue & 3.77 & 3,54 & $=\mathbf{0 . 0 1 6}$ \\
$\quad$ Donor number $\times$ Disturbance cue & 3.09 & 3,54 & $=\mathbf{0 . 0 3 4}$ \\
Rainbow trout & & & $=0.43$ \\
$\quad$ Donor number & 0.93 & 3,54 & $<0.001$ \\
$\quad$ Disturbance cue & 12.34 & 3,54 & $=0.12$ \\
$\quad$ Donor number $\times$ Disturbance cue & 1.99 & 3,54 & \\
\hline
\end{tabular}



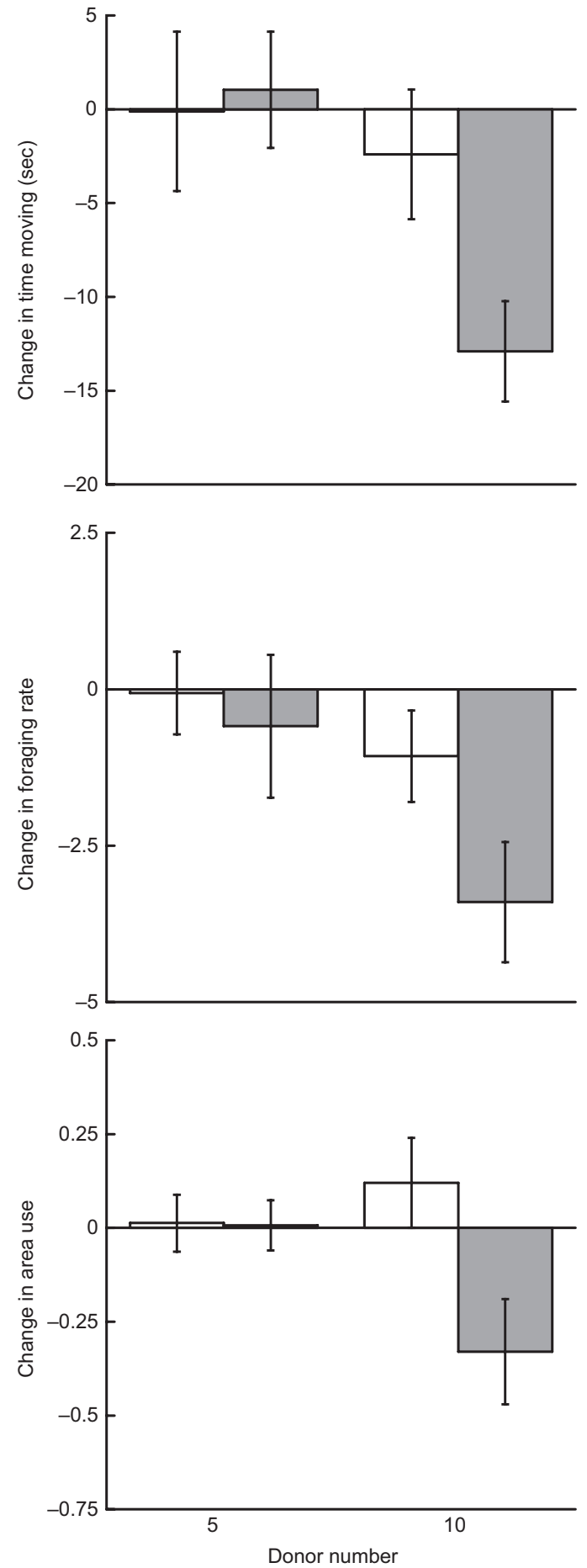

Fig. 1. Mean \pm SE change in time spent moving, foraging rate and area use for juvenile convict cichlids exposed to the odour of undisturbed conspecifics (open bars) and disturbance cues (grey bars) collected from groups of five or ten conspecific donors. $N=15$ for each treatment combination.
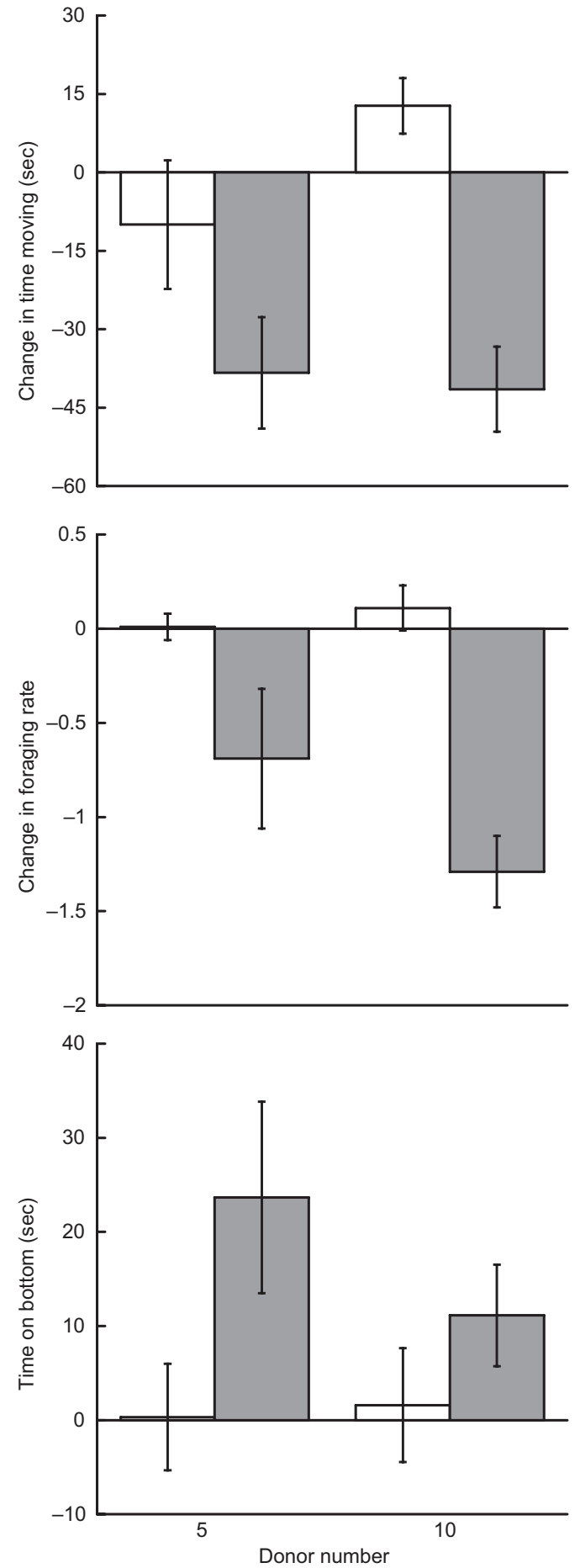

Fig. 2. Mean \pm SE change in time spent moving, foraging rate and time spent on substrate for juvenile rainbow trout exposed to the odour of undisturbed conspecifics (open bars) and disturbance cues (grey bars) collected from groups of five or ten conspecific donors. $N=15$ for each treatment combination. 

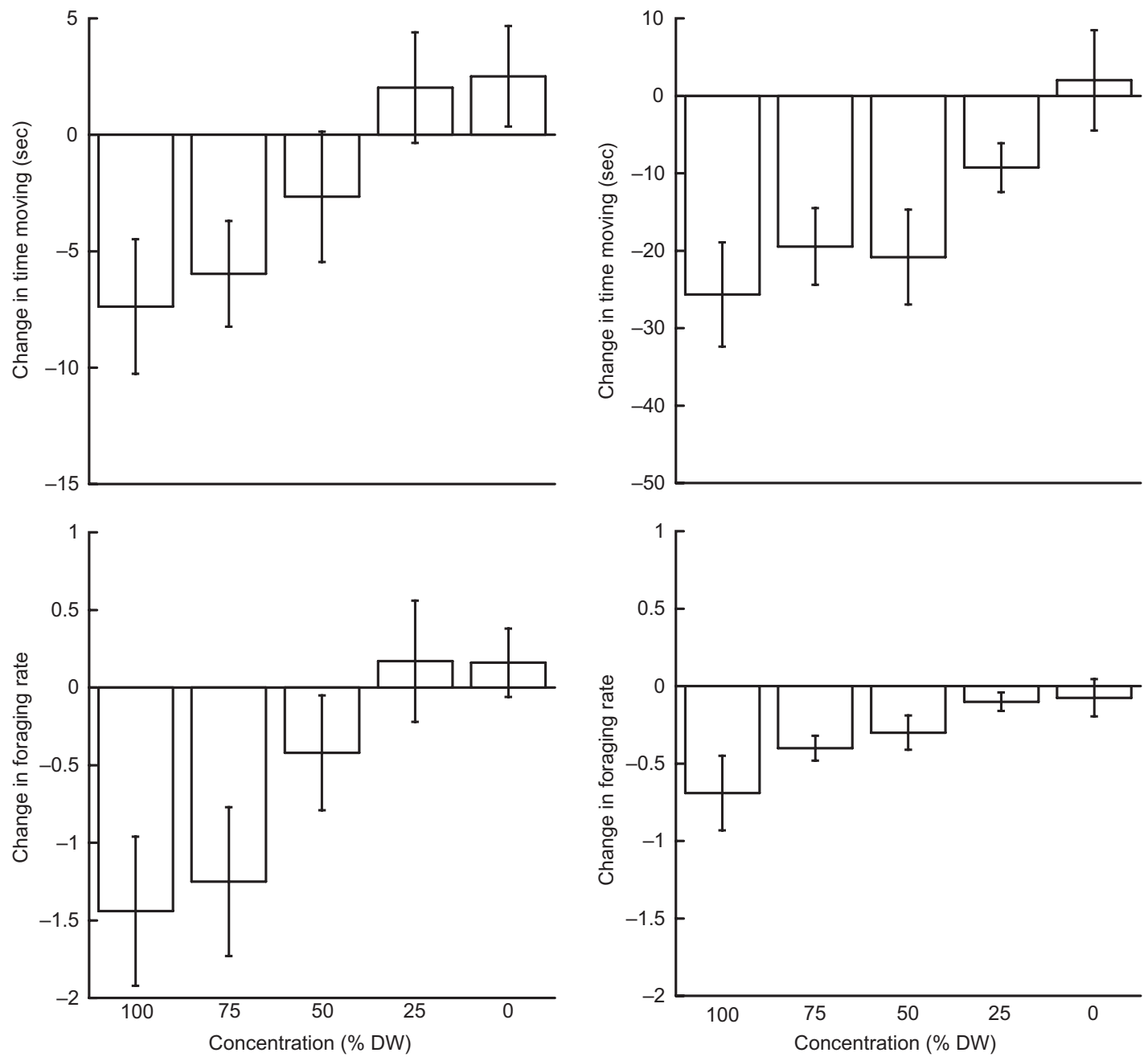

Fig. 3. Mean \pm SE change in time spent moving and foraging rate for juvenile convict cichlids exposed to varying concentrations of conspecific disturbance cues (see text for details). $N=30$ for all, except $N=29$ for $100 \%$ treatment.

Fig. 4. Mean \pm SE change in time spent moving and foraging rate for juvenile rainbow trout exposed to varying concentrations of conspecific disturbance cues (see text for details). $N=15$ for all treatments.

Table 2. Planned contrast values for linear and quadratic estimates for convict cichlids and rainbow trout exposed to varying concentrations of conspecific disturbance cues. Significant linear terms suggest graded response pattern, significant quadratic terms suggest nongraded or hypersensitive response pattern.

\begin{tabular}{clccc}
\hline & Contrast difference & $95 \% \mathrm{Cl}$ & $P$ \\
\hline Convict cichlids & & & & \\
Time moving & Linear & 8.74 & $\mathbf{3}$ & $\mathbf{0 . 0 0 1}$ \\
& Quadratic & -0.12 & $-5.09-4.86$ & $=0.96$ \\
Foraging rate & Linear & 1.47 & $0.67-2.27$ & $<\mathbf{0 0 1}$ \\
& Quadratic & -0.17 & $-0.96-0.62$ & $=0.67$ \\
Rainbow trout & & & & $<\mathbf{0 0 1}$ \\
Time moving & Linear & 20.69 & $9.45-31.93$ & $=0.28$ \\
& Quadratic & 6.14 & $-5.07-17.41$ & $=\mathbf{0 0 1}$ \\
Foraging rate & Linear & 0.48 & $0.20-0.76$ & $=0.42$ \\
& Quadratic & -0.11 & $-0.39-0.17$ & \\
\hline
\end{tabular}


concentration dependant threat-senstive response pattern. Similar response patterns are well documented for prey fishes in response to damage released chemical alarm cues (Dupuch et al. 2004, Brown et al. 2006) and learned predator recognition cues (Kusch et al. 2004, Ferrari \& Chivers 2006). These results add to our understanding of the critical importance of chemosensory cues in the threat-sensitive decision making processes of aquatic prey.

While we cannot directly compare the cichlid and trout results, as the biomass of stimulus donors differed between the species, it is interesting that both show similar overall graded response patterns. Several researchers have demonstrated considerable interspecific variability in the response pattern to varying concentrations of damage released alarm cues, ranging from graded (i.e., Jachner \& Rydz 2000, Dupuch et al. 2004) to hypersensitive responses (i.e., Marcus \& Brown 2003, Mirza \& Chivers 2003). However, Brown et al. (2006) demonstrated that juvenile convict cichlids exhibit clear graded respsones when tested in shoals, but show hypersensitive responses when tested as solitary individuals. They argued that dynamic threat-sensitive decisions would favour a graded response pattern under conditions of lower overall perceived risk and a non-graded, hypersensitive response at a higher overall levels of risk. Our current findings of a graded response towards disturbance cues is consistent with the suggestion that disturbance cues represent an overall lower relative risk as compared with damage-released cues (sensu Wisenden et al. 1995). As such, our results are in agreement with the dynamic threat-sensitive trade-off model (Brown et al. 2006).

The absolute concentration of disturbance cues needed to elicit an antipredator response is likely highly context dependent. Given that disturbance cues may be comprised of a nitrogenous waste product (Hazlett 1990a, 1990b, Keisecker et al. 1999), background levels of such compounds would dictate how much cue is required to actually elicit a response. Rather than respond to a specific behavioural response threshold (Brown et al. 2001), prey likely respond to an increase disturbance cues above background levels. Previous studies with invertebrates and amphibians suggest the disturbance cue may be ammonia(um) ions released in urine or as respiratory byproducts (Hazlett 1990a, Keisecker et al. 1999). However, recent work has failed to confirm this in juvenile cichlids and rainbow trout (M. A. Vavrek \& G. E. Brown unpubl. data). This does not preclude the possiblity that other nitrogenous waste products function as disturbance cues in prey fishes. For example, freshwater telosts excrete a small proportion of their nitrogenous waste as urea (Wilkie 2002). Alternatively, the disturbance cue may be related to stress hormones such as cortisol (Olivotto et al. 2002, Jordão 2004). Clearly additional work is needed to chemically characterize the disturbance cues in prey fishes.

Damage-released chemical alarm cues appear to be highly conserved within taxanomic groups (Mirza \& Chivers 2001b, Brown et al. 2003, Kelly et al. 2006). However, as phylogenetic distance increases, the response to heterospecific alarm cues decreases. For example, damagereleased alarm cues are highly conserved within both Ostariophsans (Brown et al. 2003) and salmonids (Mirza \& Chivers 2001b), but they do not respond to each others alarm cues (Brown et al. 2003) in the absence of learned responses (Brown 2003, Pollock et al. 2003). Vavrek (2007) has shown that juvenile convict cichlids and rainbow trout exhibit increased antipredator behaviour when exposed to each other's disturbance cues. Similarily, Hazlett (1989, 1990a) has demonstrated such a 'cross-species' response in crayfish. This observation of cross-species responses further supports the disturbance cue as a 'metabolic waste' hypothesis. If disturbance cues are indeed some generalized cue, the metabolic waste hypothesis would predict that prey guild members foraging on similar food items should produce disturbance cues that are readily recognized by distantly related prey guild members. Thus, we might expect strong selection to favour the response to pulses of disturbance cues, regardless of donor species.

To date, the studies examining distrubance cues in vertebrate and invertebrate taxa have been confined to the laboratory. Laboratory studies typically 'overestimate' the response to standardized predation cues (Lima \& Bednekoff 1999) and thus may suffer from a lack of ecological realism (Leduc et al. 2007). Thus 
despite a growing body of laboratory findings, it remains unknown if prey indivdiuals are capable of detecting and responding to disturbance cues under fully natural conditions. Clearly, field verification of the presence and use of disturbance cues is required.

In summary, our results confirm the existence of disturbance cues in both juvenile convict cichlids and rainbow trout. Moreover, our results demonstrate that both cichlids and trout exhibit graded threat-senstive responses to varying concentrations of conspecific disturbance cues. Presumably, under natural conditions, prey would be exposed to both disturbance and damagereleased chemical cues. If, as suggested by Wisenden et al. (1995), disturbance cues act as an 'early warning' cue, prey detecting a damagereleased cue following a disturbance cue should respond with a higher intensity. As such, there should be strong selection pressure on receivers to respond to all available information in order to maximize threat-sensitive trade-offs. Thus, these results add to our understanding of the critical importance of chemosensory risk assessment among aquatic prey species.

\section{Acknowledgments}

We thank Brenna Belland, Sandra Moulin and Sean Bridgeman for assistance in the laboratory and James Grant, Robert Weladji, Paul Albert and Matthew Vavrek for helpful comments on earlier versions of this manuscript. Financial support was provided by Concordia University and the Natural Sciences and Engineering Research Council of Canada to GEB. All work reported herein was conducted in accordance with Concordia University Animal Research Ethics Committee protocol \#AREC-2005-BROW.

\section{References}

Blanchet, S., Bernatchez, L. \& Dodson, J. J. 2007: Behavioural and growth responses of a territorial fish (Atlantic salmon, Salmo salar, L.) to multiple predatory cues. - Ethology 113: 1061-1072.

Brown, G. E. 2003: Learning about danger: chemical alarm cues and local risk assessment in prey fishes. - Fish and Fisheries 4: 227-234.

Brown, G. E., Adrian, J. C. Jr. \& Shih, M. L. 2001: Behavioural responses of fathead minnows (Pimephales promelas) to hypoxanthine-3- $N$-oxide at varying concentrations. - Journal of Fish Biology 58: 1465-1470.
Brown, G. E., Adrian, J. C. Jr., Naderi, N. T., Harvey, M. C. \& Kelly, J. M. 2003: Nitrogen oxides elicit antipredator responses in juvenile channel catfish, but not in convict cichlids or rainbow trout: conservation of the Ostariophysan alarm pheromone. - Journal of Chemical Ecology 29: 1781-1796.

Brown, G. E., Bongiorno, T., DiCapua, D. M., Ivan, L. I. \& Roh, E. 2006: Effects of group size on the threat-sensitive response to varying concentrations of chemical alarm cues by juvenile convict cichlids. - Canadian Journal of Zoology 84: 1-8.

Bryer, P. J., Mirza, R. S. \& Chivers, D. P. 2001: Chemosensory assessment of predation risk by slimy sculpins (Cottus cognatus): responses to alarm, disturbance, and predator cues. - Journal of Chemical Ecology 27: 533-546.

Chivers, D. P. \& Smith, R. J. F. 1998: Chemical alarm signalling in aquatic predator-prey systems: a review and prospectus. - Écoscience 5: 338-352.

Dupuch, A., Magnan, P. \& Dill, L. M. 2004: Sensitivity of northern redbelly dace, Phoxinus eos, to chemical alarm cues. - Canadian Journal of Zoology 82: 407-415.

Ferrari, M. C. O. \& Chivers, D. P. 2006: Learning threatsensitive predator avoidance: how do fathead minnows incorporate conflicting information? - Animal Behaviour 71: 19-26.

Ferrari, M. C. O., Trowell, J. J., Brown, G. E. \& Chivers, D. P. 2005: The role of learning in the development of threat-sensitive predator avoidance by fathead minnows. - Animal Behaviour 70: 777-784.

Foam, P. E., Harvey, M. C., Mirza, R. S. \& Brown, G. E. 2005: Heads up: juvenile convict cichlids switch to threat-sensitive foraging tactics based on chemosensory information. - Animal Behaviour 70: 601-607.

Godin, J.-G. J. \& Smith, S. A. 1988: A fitness cost of foraging in the guppy. - Nature 333: 69-71.

Grant, J. W. A., Girard, I. L., Breau, C. \& Weir, L. K. 2002: Influence of food abundance on competitive aggression in juvenile convict cichlids. - Animal Behaviour 63: 323-330.

Hazlett, B. A. 1989: Additional sources of disturbance pheromone affecting the crayfish Orconectes virilis. Journal of Chemical Ecology 15: 381-385.

Hazlett, B. A. 1990a: Source and nature of disturbancechemical system in crayfish. - Journal of Chemical Ecology 16: 2263-2275.

Hazlett, B. A. 1990b: Disturbance pheromone in the hermit crab Calcinus laevimanus (Randall, 1840). - Crustaceana 58: 314-316.

Helfman, G. S. 1989: Threat-sensitive predator avoidance in damselfish-trumpetfish interactions. - Behavioral Ecology and Sociobiology 24: 47-58.

Helfman, G. S. \& Winkleman, D. L. 1997: Threat sensitivity in bicolor damselfish: effects of sociality and body size. - Ethology 103: 369-383.

Jachner, A. \& Rydz, M. A. 2002: Behavioural response of roach (Cyprinidae) to different doses of chemical alarm cues (Schreckstoff). - Archiv für Hydrobiologie 155: 369-381.

Jordão, L. C. 2004: Disturbance chemical cues determine 
changes in spatial occupation by the convict cichlid Archocentrus nigrofasciatus. - Behavioral Processes 67: 453-459.

Jordão, L. C. \& Volpato, G. L. 2000: Chemical transfer of warning information in non-injured fish. - Behaviour 137: 681-690.

Kats, L. B. \& Dill, L. M. 1998: The scent of death: chemosensory assessment of predation risk by prey animals. - Écoscience 5: 361-394.

Kelly, J. M., Adrian, J. C. Jr. \& Brown, G. E. 2006: Can the ratio of aromatic skeletons explain cross-species responses within evolutionarily conserved Ostariophysan alarm cues?: testing the purine-ratio hypothesis. - Chemoecology 16: 93-96.

Kiesecker, J. M., Chivers, D. P., Marco, A., Quilchano, C., Anderson, M. T. \& Blaustein, A. R. 1999: Identification of a disturbance signal in larval red-legged frogs, Rana aurora. - Animal Behaviour 57: 1295-1300.

Kusch, R. C., Mirza, R. S. \& Chivers, D. P. 2004: Making sense of predator scents: investigating the sophistication of predator assessment abilities of fathead minnows. - Behavioral Ecology and Sociobiology 55: 551-555.

Leduc, A. O. H. C., Roh, E., Breau, C. \& Brown, G. E. 2007: Learned recognition of a novel odour by wild juvenile Atlantic salmon, Salmo salar, under fully natural conditions. - Animal Behaviour 73: 471-477.

Lima, S. L. \& Dill, L. M. 1990: Behavioral decisions made under the risk of predation: a review and prospectus. - Canadian Journal of Zoology 68: 619-640.

Lima, S. L. \& Bednekoff, P. A. 1999: Temporal variation in danger drives antipredator behavior: the predation risk allocation hypothesis. - American Naturalist 153: 649-659.

Marcus, J. M. \& Brown, G. E. 2003: Response of pumpkinseed sunfish to conspecific chemical alarm cues: an interaction between ontogeny and stimulus concentration. - Canadian Journal of Zoology 81: 1671-1677.

Mirza, R. S. \& Chivers, D. P. 2000: Predator-recognition training enhances survival of brook trout: evidence from laboratory and field-enclosure studies. - Canadian Journal of Zoology 78: 2198-2208.
Mirza, R. S. \& Chivers, D. P. 2001a: Chemical alarm signals enhance survival of brook charr (Salvelinus fontinalis) during encounters with predator chain pickerel (Esox niger). - Ethology 107: 989-1005.

Mirza, R. S. \& Chivers, D. P. 2001b: Are chemical alarm cues conserved within salmonid fishes? - Journal of Chemical Ecology 27: 1641-1655.

Mirza, R. S. \& Chivers, D. P. 2003: Response of juvenile rainbow trout to varying concentrations of chemical alarm cue: response thresholds and survival during encounters with predators. - Canadian Journal of Zoology 81: 88-95.

Nishizaki, M. T. \& Ackerman, J. D. 2005: A secondary chemical cue facilitates juvenile-adult postsettlement associations in red sea urchins. - Limnology and Oceanography 50: 354-362.

Olivotto, I., Mosconi, G., Maradonna, F., Cardinali, M. \& Carnevali, O. 2002: Diplodus sargus interregnalpituitary response: chemical communication in stressed fish. - General and Comparative Endocrinology 127: 66-70.

Pollock, M. S., Chivers, D. P., Mirza, R. S. \& Wisenden, B. D. 2003: Fathead minnows, Pimephales promelas, learn to recognize chemical alarm cues of introduced brook stickleback, Culaea inconstans. - Environmental Biology of Fishes 66: 313-319.

Vavrek, M. A. 2007: Disturbance cues in prey fishes: an additional source of chemosensory risk assessment. M.Sc. thesis, Concordia University, Montreal, Quebec, Canada.

Wilkie, M. P. 2002: Ammonia excretion and urea handling by fish gills: present understanding and future research challenges. - Journal of Experimental Zoology. 293: 284-301.

Wisenden, B. D. 2000: Olfactory assessment of predation risk in the aquatic environment. - Philosophical Transactions of the Royal Society of London B 355: 1205-1208.

Wisenden, B. D., Chivers, D. P. \& Smith, R. J. F. 1995: Early warning in the predation sequence: a disturbance pheromone in Iowa darters (Etheostoma exile). - Journal of Chemical Ecology 21: 1469-1480. 